AJChE 2014, Vol. 14, No. 2, 38 - 47

\title{
A Morphological Study of
}

\section{Poly [Vinylidene Fluoride] Pudf Membranes:}

\section{In Perspective of}

\section{Membrane Pervaporation Process}

\author{
Adil Hatem Rashid ${ }^{*}, 1,2$ \\ M. D. Irfan Hatem ${ }^{1}$
}

Muhammad Syarhabil Ahmad ${ }^{1}$

Mohd. Hafiz Dzarfan Othman ${ }^{3}$

1 School of Bioprocess Engineering. Universiti Malaysia Perlis. Malaysia. kompleks pusat pengajian, Jejawi 3, 02600 Arau, Perlis, Malaysia

${ }^{2}$ Faculty of Chemical Engineering. Al-Muthanna University. Alsamawah. Iraq

${ }^{3}$ Advanced Membrane Technology Research Centre (AMTEC), Faculty of Petroleum and Renewable Energy Engineerign. Universiti Teknologi Malaysia, 81310 UTM Johor Bahru, Malaysia.

"e-mail : adil.hatem@yahoo.com

Membrane separation plays an important role in chemical industry (Prabhat et al. 2011). Pervaporation is one of the most promising membrane separation processes which is used in a wide range of applications such as separation of organic mixtures and dehydration of solvents.

This study investigated the effect of polymer concentration on the morphology of pervaporation membrane, where polyvinylidene fluoride (PVDF) was chosen as the base polymeric material while phase inversion method was employed as a technique to prepare an asymmetric PVDF membrane. The polymer concentration in dope solution was varied from 16 to $20 \mathrm{wt} \%$ and the prepared membranes were characterized in terms of its structure, porosity, contact angle, and pore size. Five PVDF membrane samples were prepared (without any post-treatment) with polymer concentration of $16,17,18,19$, and $20 \mathrm{wt} \%$ respectively. Depending on permeating component, two main areas of pervaporation processes can be identified: hydrophobic and hydrophilic and consequently two types of membranes could be observed: hydrophobic and hydrophilic membranes.

From the results, it was found that the increase in polymer concentration has produced membranes with low pore size and porosity and as a result, high contact angle (low hydrophilicity).The highest contact angle of $83^{\circ}$ was found for membrane with highest polymer concentration of $20 \mathrm{wt} \%$ with the lowest pore size and porosity of $(0.4156 \mu \mathrm{m}$ and $65 \%)$ respectively. The lowest contact angle of $76^{\circ}$ was found for membrane with the lowest polymer concentration of $16 \mathrm{wt} \%$ with the highest pore size and porosity $(0.8671 \mu \mathrm{m}$ and78\%) respectively.

Keywords : Membrane separation, Pervaporation (PV), PVDF membrane, Phase inversion, Polymer Concentration, Membrane morphology. 


\section{INTRODUCTION}

Due to its significant advantages over the other separation processes, membrane separation plays an important role in chemical industry. In comparison to other separation technologies, membranes offer lower energy consumption, cheaper construction cost and higher selectivity (Prabhat et al. 2011). There are various types of membrane processes one of which is pervaporation, which is used in a wide range of applications such as separation of organic mixtures and dehydration of solvents (Jinhui et al. 2013)..

Pervaporation is a separation process in which a liquid mixture contacts the membrane, which preferentially permeates one of the liquid components as a vapor. The vapor, which is enriched in the more permeable component, is cooled and condensed, spontaneously generating a vacuum that drives the process (Johanna et al. 2013). Membrane materials and fabrication method are among the key factors that determine the performance of pervaporation membrane. Compared to other separation processes, such as distillation and evaporation, it offers lower energy and often is refered to as (clean technology); especially for the treatment of volatile organic compound. Thus it is considered a promising alternative to these processes. Different from thermal processes, the separation in PV process is not based on relative, but rather on the relative rates of permeation through a membrane (Enu et al. 2011).

Poly(vinylidene fluoride) (PVDF) has been considered as a very attractive polymer as well as membrane material, exhibiting superior mechanical, thermal, and chemical properties, and is widely used to synthesize a lot of membrane separations (Mônica et al. 2012). For different types of applications such as pervaporation, membrane distillation, and others. Thus, it is the best option of membrane material (Masuelli et al. 2012). First fluorinated polymeric PVDF membrane was fabricated early 1980s (Fu Liu et al. 2011). Phase inversion, stretching, electrospining, track-etching, and interfacial polymerization are the most commercially employed techniques for membranes fabrication.

Due to its simplicity and flexible production scale, phase inversion method is used to produce the most of the commercial membranes (Boor et al. 2013). Phase inversion is a process where a polymer is transferred from a liquid to solid phase (Yee et al. 2011). Preparation of phase inversion membranes often follows five different techniques which are precipitation by solvent evaporation, precipitation from vapor phase, precipitation by controlled evaporation, thermal precipitation, and immersion precipitation (Agnieszka et al. 2013). The most commercial polymeric membranes are produced by immersion precipitation. In immersion precipitation process the polymer solution (polymer + solvent) is cast on a suitable support followed by immersion in a coagulation bath (nonsolvent) and as a result of the exchange of solvent and nonsolvent the precipitation occurs (Iwona et al. 2011).

An ideal pervaporation membrane should consist of an ultra-thin defect free 
dense layer (skin layer) supported by a porous support (asymmetric structure). The skin layer is perm-selective and hence responsible for the selectivity of the membrane. Industrially, asymmetric structure is the most popular and the phase inversion is the common method to fabricate asymmetric membranes (Wang and Lai 2013).

In this method, two phase systems that are produced from a polymer solution transformation, consist of polymer poorliquid phase which form the voids and the polymer rich-solid phase which forms the rigid membrane structure (Agnieszka et al. 2013). Many parameters such as solvent type, evaporation time, coagulation bath medium and temperature, non-solvent additives, and polymer concentration could give significant effects on PVDF membrane morphologies and eventually the membrane performance (Fu Liu et al. 2011).

The objective of this study is to verify the effect of the polymer concentration on the membrane morphologies in terms of its structure, contact angle, porosity, and pore size.

\section{EXPERIMENTAL}

\section{Material and Methods}

\section{Materials}

The materials used for fabrication included PVDF (average Mwt 534,000 by PC, powder) (Sigma Aldrich) and (1Methyl-2-Pyrrolidinone

(NMP)) (Anhydrous 99.5\%) (Sigma Aldrich) as a solvent.

\section{Preparation Method}

Poly(vinylidene fluoride) (PVDF) powder was dissolved in (1-Methyl-2Pyrrolidinone) (NMP) with a determined polymer concentration (16-20wt\%) and a dissolution temperature $\left(30-40^{\circ} \mathrm{C}\right)$ for $6 \mathrm{~h}$. This was followed by cooling at room temperature for $3 \mathrm{~h}$ to remove all bubbles formed upon dissolution. After $3 \mathrm{~h}$ the solution was cast on a glass plate with a certain thickness of $250 \mu \mathrm{m}$ followed by immersion of the cast film with the glass plate in the coagulation bath until the membrane formed. The formed membrane then was left immersed in the coagulation bath for $24 \mathrm{~h}$ followed by drying at room temperature for another $24 \mathrm{~h}$ before characterization. Five flat sheet PVDF polymeric membranes samples with different polymer concentration in polymer solution $(16,17,18,19$, and 20wt\%) were prepared (without any posttreatment) at dissolution temperature of $40^{\circ} \mathrm{C}$. Deionized water (100\%) was used as a coagulation medium to remove all the residual solvent as well as to complete the membrane solidity. The composition and preparation conditions of the prepared membranes are shown in Table 1.

\section{Characterization of Prepared Membranes}

\section{SEM Microscopy}

The morphology of the prepared PVDF membranes was observed using scanning electron microscope (SEM) (Hitachi TM3000). The prepared membranes were coated with platinum using Auto line cutter, JEOL: JFC-1600 to reduce sample charging under the electron beam. 
Table 1. Compostition and preparation conditions of prepared membranes

\begin{tabular}{cccccc}
\hline Membrane & $\begin{array}{c}\text { Polymer } \\
\text { Concentration } \\
\text { wt \% }\end{array}$ & $\begin{array}{c}\text { Solvent } \\
\text { Concentration } \\
\text { wt \% }\end{array}$ & $\begin{array}{c}\text { Dissolution } \\
\text { Temp. }\left({ }^{\mathbf{0}} \mathbf{C}\right)\end{array}$ & $\begin{array}{c}\text { Dissolution } \\
\text { Time (h) }\end{array}$ & $\begin{array}{c}\text { Coagulation } \\
\text { Medium }\end{array}$ \\
\hline M1 & 16 & 84 & 40 & 6 & $100 \%$ water \\
M2 & 17 & 83 & 40 & 6 & $100 \%$ water \\
M3 & 18 & 82 & 40 & 6 & $100 \%$ water \\
M4 & 19 & 81 & 40 & 6 & $100 \%$ water \\
M5 & 20 & 80 & 40 & 6 & $100 \%$ water \\
\hline
\end{tabular}

\section{Contact Angle Measurement}

Contact angle measurements on the prepared membranes were conducted using the contact angle goniometer (Model: OCA 15EC, Dataphysics with deionized water as contact liquid. The water droplets of $2 \mu \mathrm{L}$ were dropped on the membrane surfaces. An average and standard deviation of at least 5 independent measurements was obtained at different points of one sample.

\section{Porosity}

To measure the prepared membranes porosity, PVDF membranes were immersed in octanol (ACS, ISO, Reag Ph Eur, Merck) for 2 hours and then the membrane surface was dried by filter paper. The wet and dry membranes were weighed then the porosity was calculated using Eq (1) [5]:

$$
\varepsilon=\frac{\left(\frac{m_{b}}{\rho_{b}}\right)}{\left(\frac{m_{b}}{\rho_{b}}+\frac{m_{p}}{\rho_{p}}\right)} \times 100
$$

where $\varepsilon$ is the porosity of membrane (\%), $m_{b}$ is the mass of the observed octanol $(\mathrm{gm}), \mathrm{m}_{\mathrm{p}}$ is the mass of dry membrane (gm) $\mathrm{pb}$ is the density of octanol $\left(\mathrm{gm} / \mathrm{cm}^{3}\right)$ $\rho_{b}$ is the density of octanol $\left(\mathrm{gm} / \mathrm{cm}^{3}\right)$ and $\rho_{p}$ is the density of membrane $\left(\mathrm{gm} / \mathrm{cm}^{3}\right)$.

\section{Pore Size Measurement}

Pore size distribution of the prepared membranes was measured by using a Porolux 1000 Porometer (IB-FT GmbH, Germany).

\section{RESULTS AND DISCUSSION}

In order to investigate the role of polymer concentration on membrane morphologies and performances, different polymer concentration (16-20wt\%) were used. The membrane composition and preparation conditions are shown in Table 1. The effect of polymer concentration on the membrane morphology is discussed in this study:

\section{Surface and Cross Section Morphology}

Fig.1. shows the surface morphology of the prepared membranes (M1, M2, M3, M4, and M5).The images clearly show the effect of the increase in the polymer concentration on membrane surface. It was shown that for the membranes with higher polymer concentration a dense skin layer with very small pores was observed while a dense skin layer exhibited larger 

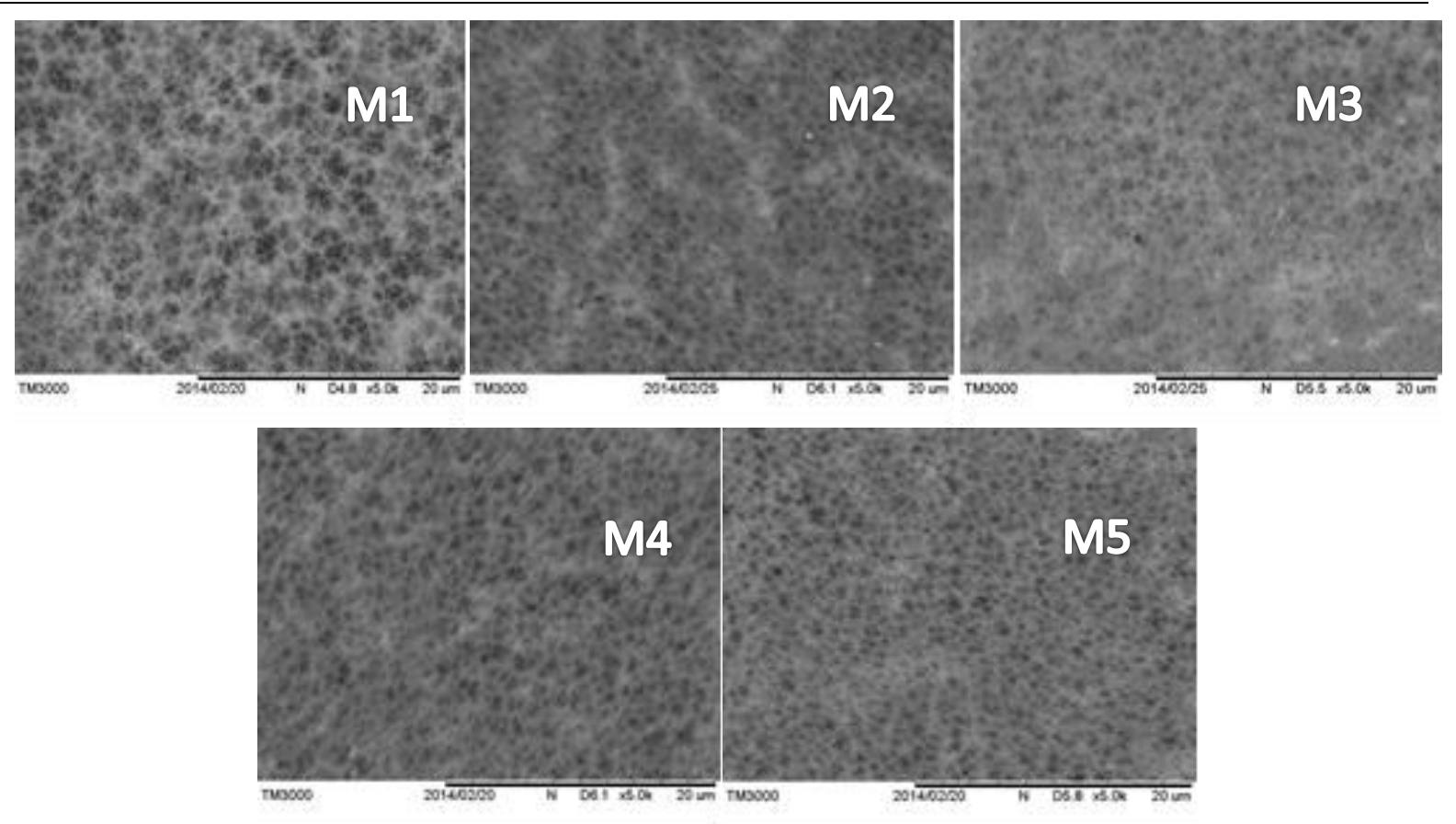

Fig. 1: SEM images for membranes surface (M1, M2, M3, M4,and M5), (Top surface).

pores for membranes with lower concentration in the order of $\mathrm{M} 1>\mathrm{M} 2>\mathrm{M} 3>\mathrm{M} 4>\mathrm{M} 5$ with values of $(0.8671, \quad 0.7155,0.6454,0.5991$, and $0.4156)$ respectively.

It was also shown that the number of pores is higher for membranes with lower concentration in comparison to those with higher concentration $(\mathrm{M} 1>\mathrm{M} 2>\mathrm{M} 3>\mathrm{M} 4>\mathrm{M} 5)$. With the increase in polymer concentration of the solution, coagulation value was reduced. That could be attributed to stronger polymer/solvent interaction and slower interaction between nonsolvent and polymer, which lowered down the solvent power for polymer. Slower precipitation rate could have resulted in the formation of a skin layer and, then, the diffusional exchange between solvent (NMP) and nonsolvent (water) in the sublayer would be hindered (Andy et al. 2013). Depending on the rate of polymer precipitation, fast phase separation at surface layer and slower phase separation at sublayer resulted in asymmetric membrane formation with dense and thick skin layer by a higher polymer concentration solution (as shown in Fig.2) (Rinku et al. 2014). Also, as shown in Fig.2 all prepared membranes have an asymmetric structure consisting a dense top layer and a porous sublayer. The number of macrovoids was decreased with the increase of polymer concentration and the finger like structure was clearly shown in the cross section of membranes with lower polymer concentration (M1 and $\mathrm{M} 2$ ) of (16 and $17 \mathrm{wt} \%$ ) respectively. It was noted that hydrophilic pervaporation processes require a hydrophilic pervaporation membranes with higher pore size and porosity and as a result lower contact angle $\left(<90^{\circ}\right)$. At the same time hydrophobic pervaporation membranes with lower pore size and porosity, and as a result higher contact 


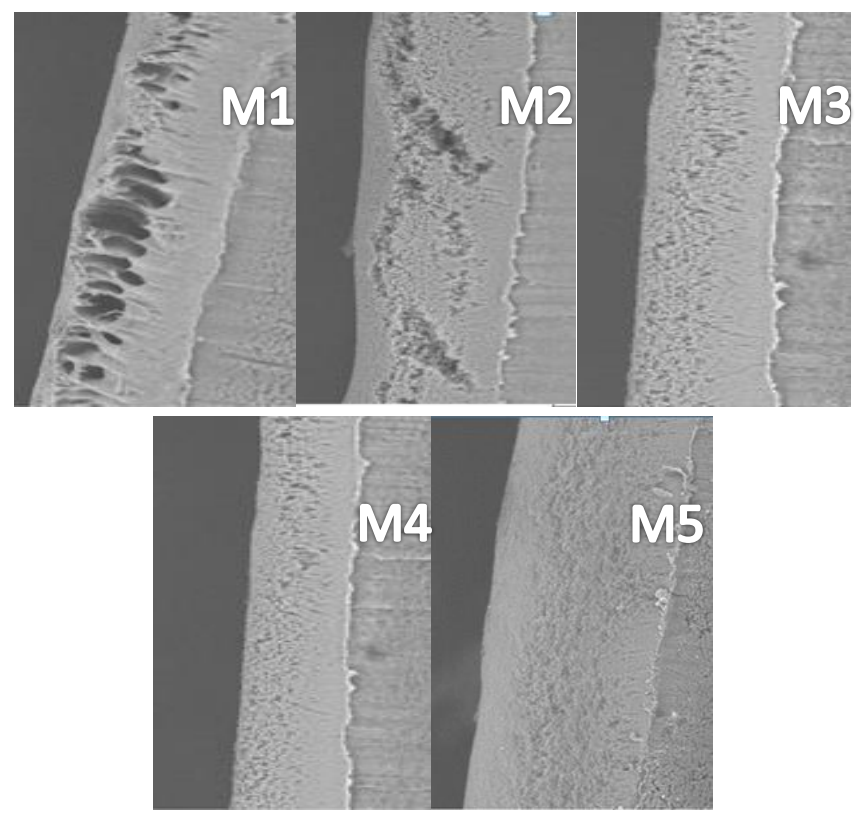

Fig. 2: SEM cross-sectional images of PVDF membranes (M1, M2, M3, M4, and M5)

angle $\left(\geq 90^{\circ}\right)$, are required to hydrophobic pervaporation processes.

\section{Porosity and Pore Size}

Porosity or void fraction is a measure of the void spaces in a material, and is a fraction of the volume of voids over the total volume, between 0 and 1 , or as a percentage between 0 and $100 \%$.

The prepared membranes in our work were also characterized in terms of porosity and pore size measurement. Based on the results shown in Figs.3(a) and (b), pore size and porosity values ('0.8671, 0.7155, 0.6454, 0.5991, and $0.4156 \mu \mathrm{m})$ and $(78 \%, 75.5 \%, 72 \%, 69 \%$, and $65 \%$ respectively exhibited a declining trend with increasing polymer concentration in dope solution (M5 $<\mathrm{M} 4<\mathrm{M} 3<\mathrm{M} 2<\mathrm{M} 1)$ of $(20,19,18,17$, and $16 \mathrm{wt} \%)$ respectively.That which proved the proportional relationship between the pore size and porosity while the reverse relationship of the polymer concentration with both of pore size and porosity was clear. From the results, the highest values of pore size $(0.8671 \mu \mathrm{m})$ and porosity (78\%) were found for membrane with the lowest polymer concentration of (16 wt\% ) while the lowest values of pore size $(0.4156 \mu \mathrm{m})$ and porosity $(65 \%)$ were found for membrane with the highest polymer concentration of (20wt\%).

Lower porosity for higher polymer concentration could be attributed to the slower exchanging process between the solvent and non-solvent which resulted in a dense and compact membrane structure (Ahmad, and Ramli 2013).

As stated before, the increase of the polymer concentration in polymer solution leads to a lower diffusion rate of nonsolvent and as a result leads to a higher mass transfer resistance between the nonsolvent and solvent in the system during solidification of the casting solution. Thus, denser membrane with smaller pore size distribution will be formed after a long 
44 A Morphological Study of Poly (Vinylidene Fluoride) Pvdf Membranes:In Perspective of Membrane Pervaporation Process
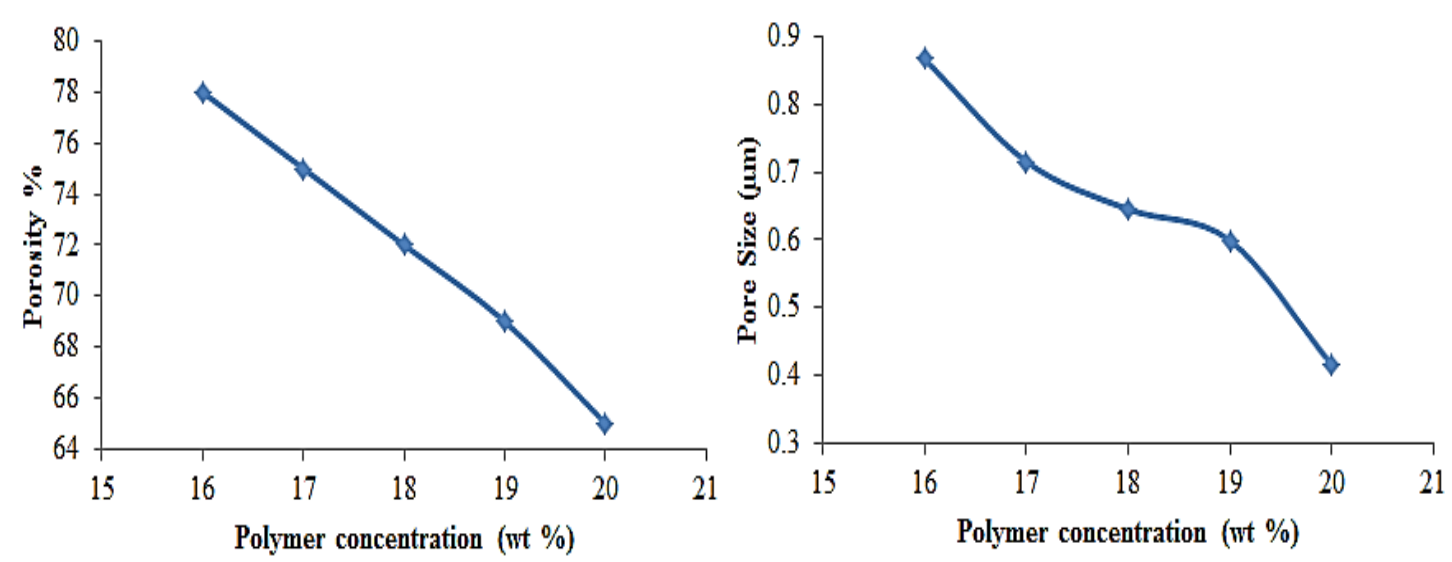

Fig. 3: Porosity and pore size as a function of polymer concentration.(a) Porosity; (b) Pore size.

period of precipitation process (Ahmed et al. 2013).

Hydrophilic pervaporation processes require a hydrophilic pervaporation membranes with higher pore size and porosity. At the same time, hydrophobic pervaporation membranes with lower pore size and porosity are required to hydrophobic pervaporation processes The results were also supported with SEM images of membranes presented in Figs. 1 and 2.

\section{Contact Angle}

In chemistry, the repelled molecules from a mass of water refer to its hydrophobicity. It is represented in terms of the contact angle between the water and the surface of the membrane The contact angle is the angle between the solid and liquid surface. When water is applied to the surface, the outermost surface layers interact with the water and the membrane is said to be hydrophobic if the contact angle exceeds $90^{\circ}$. A high contact angle could be achieved on a hydrophobic surface with low free energy whereas a wet high energy allows the drop to spread resulting in a low contact angle (Ahmad et al. 2012).

Porous surfaces of the membranes have a significant effect on the hydrophobic property of PVDF membranes. In order to eliminate the wetting problems, the membranes must have high hydrophobicity at the same time low hydrophobicity combined with bigger pore size. A membrane with a higher polymer concentration has higher resistance for the water to flow mainly due to the smaller pore size as well as an increased higher contact angles (Ooi et al. 2012).

As shown in Figs. 3(a) and (b) the high polymer concentration in polymer solution produced membranes with low pore sizes (0.4156, 0.5991, 0.6454, 0.7155, and $0.8671 \mu \mathrm{m})$ and low porosities of $(65 \%$, $69 \%, 72 \%, 75.5 \%$, and $78 \%$ ) in the order $(\mathrm{M} 5<\mathrm{M} 4<\mathrm{M} 3<\mathrm{M} 2<\mathrm{M} 1)$ respectively. As a result, an increase in the polymer concentration increased the contact angle values (as shown in Fig.4) $(\mathrm{M} 5>\mathrm{M} 4>\mathrm{M} 3>\mathrm{M} 2>\mathrm{M} 1)$ of $\left(83^{\circ}>81^{\circ}>\right.$ $79^{\circ}>77^{\circ}>76^{\circ}$ ) respectively (intrinsic 
contact angle of dense PVDF membrane is less than $90^{\circ}$ ) (Kang and Cao 2014).

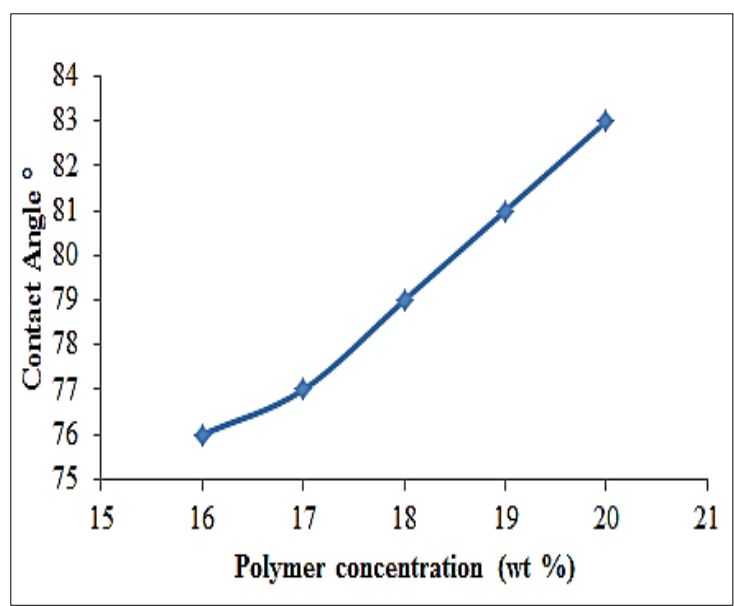

Fig. 4: Contact Angle as a function of polymer concentration

It is noted that the hydrophobicity of PVDF membranes is affected by porous surfaces of the membranes. The highest contact angle $\left(83^{\circ}\right)$ was found for membrane with the highest polymer concentration of (20wt\%) while the lowest one $\left(76^{\circ}\right)$ was found for membrane with the lowest polymer concentration of (16wt\%). This has proved the proportional relationship between the contact angle and polymer concentration and as a result the reverse relationship of the contact angle with both of pore size and porosity was observed (high polymer concentration produced a low free energy surface membrane).

As stated before, membranes with higher contact angle $\left(\geq 90^{\circ}\right)$ are required for hydrophobic pervaporation processes. On the other hand, hydrophilic pervaporation processes require hydrophilic membranes with lower contact angle $\left(<90^{\circ}\right)$.

\section{CONCLUSIONS}

Pervaporation is one of the most promising membrane separation processes which is used in a wide range of applications such as separation of organic mixtures and dehydration of solvents.

- The type of permeating component plays a very important role in pervaporation processes classification. Two main areas of PV can be identified: hydrophobic and hydrophilic PV and consequently two types of PV membranes could be observed: hydrophilic (contact angle $<90^{\circ}$ ) and hydrophobic membranes (contact angle $\geq 90^{\circ}$ ). For dehydration of solvents, hydrophilic membranes are always used while hydrophobic (or hydrophilic) membranes are used for separation of organic mixtures.

- Hydrophobicity/hydrophilicity of membranes is always affected by all of the above mentioned factors (membrane structure, pore size, and porosity). Thus, they have a significant effect on the determination of the appropriate type of membrane used in PV process.

- In this study, it was found that polymer concentration in polymer solution has a significant effect on membrane morphology in terms of its structure, pore size, porosity, and contact angle.The results showed that the polymer concentration has a reverse relationship with both of pore size $(0.8671,0.7155,0.6454,0.5991$, and $0.4156 \mu \mathrm{m})$ and porosity $(78,75.5$, 
46 A Morphological Study of Poly (Vinylidene Fluoride) Pvdf Membranes:In Perspective of Membrane Pervaporation Process

72, 69, and 65\%) for membranes (M1, $M 2, M 3, M 4$, and $M 5$ ) respectively and , as a result, the contact angle increased proportionally $\left(76^{\circ}, 77^{\circ}\right.$, $79^{\circ}, 81^{\circ}$, and $83^{\circ}$ ) with the increased polymer concentration for membranes (M1, M2, M3, M4, and M5) respectively.

- The highest value of contact angle $\left(83^{\circ}\right)$ was found for membrane with the highest polymer concentration of (M5) with lowest pore size of $(0.4156 \mu \mathrm{m})$ and lowest porosity of (65\%). In contrast, the membrane with the lowest polymer concentration (M1) produced the lowest contact angle of $\left(76^{\circ}\right)$, highest pore size of $(0.8671 \mu \mathrm{m})$, and highest porosity of (78\%).

- Experimentally, there are several approaches to improve any of the mentioned factors (membrane structure, porosity, pore size, and contact angle) depending strongly on the PV separation process type and conditions.

\section{NOMENCLATURE}

$$
\begin{array}{ll}
\varepsilon: \text { Porosity of the membrane (\%) } \\
m_{b}: \text { Mass of the observed octanol } \\
& (\mathrm{gm}) \\
\rho_{b}: \text { Density of octanol }\left(\mathrm{gm} / \mathrm{cm}^{3}\right) \\
m_{p}: \text { Mass of the dry membrane }(\mathrm{gm}) \\
\rho_{p}: \text { Density of PVDF membrane } \\
& \left(\mathrm{gm} / \mathrm{cm}^{3}\right)
\end{array}
$$

\section{REFERENCES}

1. Agnieszka, K.H.; Ben A.; Wouter S.; and Vankelecoma F.J.(2013). Study of polymer concentration and evaporation time as phase inversion parameters for polysulfone-based SRNF membranes. Journal of Membrane Science,442, 196-205.

2. Ahmad A.L., and Ramli W.(2013). Hydrophobic PVDF membrane via two-stage soft coagulation bath system for Membrane Gas Absorption of CO2. Separation and Purification Technology, 103, 230-240.

3. Ahmad A.L., Ideris N., Ooi B, Low $S$, and Ismail A. (2013). Influence of Polymer Concentration on PVDF Membrane Fabrication for Immunoassay Analysis. Journal of Applied Sciences, ISSN 1812-5654.

4. Ahmad A.I., Ramli W, Fernando W, and Daud W (2012). Effect of ethanol concentration in water coagulation bath on pore geometry of PVDF membrane for Membrane Gas Absorption application in $\mathrm{CO} 2$ removal. Separation and Purification Technology, 88, 11-18.

5. Andy C., Walter K., Yufeng Z., and Xianshe F. (2013). A study of thermodynamics and kinetics pertinent to formation of PVDF membranes by phase inversion. Desalinatio, 309, 156164.

6. Boor S., Victor K., Raed H., and Nidal H. (2013). A review on membrane fabrication: Structure, properties and performance relationship. Desalination, 326, 77-95.

7. Enu J., Albert S., and Yong T. (2011). Pervaporation of butanol/water mixtures using siloxane polymer/ceramic composite 
membranes. Desalination and water treatment, 48, Issue 1-3..

8. Fu Liu, Awanis H., Yutie L., Moghareh A., and K. Li (2011). Progress in the production and modification of PVDF membranes. Journal of Membrane Science, 375, 1-27.

9. Iwona S., Mafalda P., and Andrew L. (2011). The effect of membrane formation parameters on performance of polyimide membranes for organic solvent nanofiltration (OSN): Part A. Effect of polymer/solvent/non-solvent system choice. Journal of Membrane Science, 381, 152-162.

10. Jinhui W., Genghao G., Masakoto K., Tomohisa Y., Kenji Ito, and Toshinori T. (2013). Pervaporation performance and characterization of organosilica membranes with a tuned pore size by solid-phase $\mathrm{HCl}$ post-treatment. Journal of Membrane Science, 441, 120-128.

11. Johanna N., Wojciech K., and Riitta L.K. (2013). Pervaporation performance of composite poly(dimethyl siloxane) membrane for butanol recovery from model solutions. Journal of Membrane Science, 434, 55-64.

12. Kang G-d., and Cao Y-M (2014). Application and modification of poly(vinylidene fluoride) (PVDF) membranes: A review. Journal of Membrane Science ,463, 145-165.

13. Masuelli M.A, Grasselli M, Marchese J., and Ochoa N.A (2012). Preparation, structural and functional characterization of modified porous PVDF membranes by $\gamma$-irradiation. Journal of Membrane Science, 389, 91 98.
14. Mônica B.T., Patrícia P., Marcos M., Jocelei D., and Mara Z. (2012). Effect of non-solvents used in the coagulation bath on morphology of PVDF membranes. Material research, 15, 882-890.

15. Ooi B.S., Yatim N., Ahmad A.L., and Lai S.O.(2012). Preparation of polyvinylidene fluoride membrane via dual coagulation bath system and its wettability study. Journal of Applied Polymer Science, 124, E225-E232.

16. Prabhat G., Singh R.P., and Veena C. (2011). Pervaporation separation of organic azeotrope using poly(dimethyl siloxane)/clay nanocomposite membranes. Journal of Separation and Purification Technology , 80, 435-444.

17. Rinku T., Elena G-B, and Hassan A. (2014). Pore structure control of PVDF membranes using a 2-stage coagulation bath phase inversion process for application in membrane distillation (MD). Journal of Membrane Science, 452, 470-480.

18. Wang D-M.and Lai J-Y (2013). Recent advances in preparation and morphology control of polymeric membranes formed by nonsolvent induced phase separation. Current Opinion in Chemical Engineering, 2, 229-237.

19. Yee K., Natalia W., and Chung T-S (2011). Pervaporation of semicrystalline poly(vinylidene fluoride) membrane formation and its prospects for biofuel (ethanol and acetone) separation via pervaporation. Journal of Membrane Science, 378, 149-162. 